\title{
Preface
}

\section{Endocrine Pathology: Treading Water in a Shark Tank of Uncertain Malignant Potential}

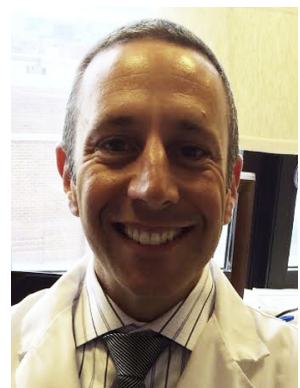

Peter M. Sadow, MD, PhD

Editor

A wonderful thing about endocrine-related diseases is that most of them make sense. Unlike the complicated molalities of the Loop of Henle or the mapping of the complex and vaguely specific geography of the brain, we can logically contemplate the mechanism of action of central to end-organ feedback of hormone regulation. If the body physiologically requires more calcium, calcium sensors tell the parathyroid to facilitate increased calcium absorption and secretion. To maintain homeostasis in body temperature and metabolism, thyrotropin-releasing hormone does a well-rehearsed dance with thyroid-stimulating hormone (TSH; or thyrotropin), which, in turn, greets the transmembrane TSH receptor in the thyroid to facilitate iodine uptake via the sodium iodide symporter and the production of thyroid hormone by thyrocytes. From thyroglobulin stores within thyroid follicles, there is secretion of thyroid hormone in its active form of triiodo-L-thyronine as well as in its primary form of thyroxine to be activated by cells in the body's periphery, resulting in intracellular modification of gene and protein expression via complexes associated with thyroid hormone receptor isoforms, and subsequently feeding back centrally to cool down additional secretion in times of plenty. For as much as we understand about endocrine physiology, a great deal is left to be discovered about endocrine pathophysiology, much of which centers around endocrine neoplasia.

Endocrine neoplasia comes in two main forms: lesions that are hormone-secreting and those that are nonsecreting. After that generalization, most other points remain rudimentary in our knowledge, especially in regard to the determination of biological behavior of these neoplasms, from incidental to low biological potential to potentially aggressive to frankly malignant.

Immunohistochemistry and more recently available molecular diagnostic tools have added layers to our documentation and understanding of gene expression profiles of endocrine neoplasms; however, for the most part, while helping us to more distinctly classify some tumor types, predicting behavior or response to particular treatment modalities is still, in many cases, elusive.

In this issue of Surgical Pathology Clinics devoted to Endocrine Pathology, we explore some emerging topics in this kinetic field as authored by several of its leaders accompanied by some rising stars. The breadth of topics includes the thyroid gland, revisiting the utility of immunohistochemistry in the diagnosis of malignancy, in addition to a thoughtful overview of the recently refined diagnosis of poorly differentiated thyroid carcinoma. Our discussion of the thyroid is rounded out by a foray into the role of the immune 
system in the regulation of thyroid malignancy. Additional lightning rods for discussion are papillary thyroid carcinomas with high-grade features and the ever-troublesome follicular variant of papillary thyroid carcinoma. As additional molecular and outcomes data emerge from these entities, increasing in frequency of diagnosis, we can then make a more compelling story as to how to best classify them.

Accompanying the thyroid articles, authors explore our current understanding of parathyroid disease, pancreatic neoplasia, adrenal cortical disease, and paragangliomas, with particular attention to practical and molecular diagnostic approaches toward these organs that produce lesions of both certain and uncertain biological potential, with modulation of treatment and follow-up depending on the tumor morphology and molecular characterization.

Finally, we give an account of a diverse group of diseases under the auspices of familial endocrine syndromes. This group of neoplasias, having at least one major component of the syndrome as an endocrine-related malady, consists of some well-known entities with long-standing cause in addition to more recently described cohorts pending more thorough characterization. Included in the description of these syndromes are hallmark features and clues for the pathologist that may aid in the diagnosis of a specific syndromic entity as well as the basis to guide our clinical colleagues toward broader exploration for associated abnormalities.

Although this issue is not exhaustive in its scope, it is my hope that the reader finds these topics to be both practical and informative. I am exceptionally thankful to the authors of these articles, who, along with their colleagues and trainees, have produced a truly thoughtful state of the field, and who make me very thankful and honored to be a member of the endocrine pathology community. I am particularly thankful for those authors who submitted their articles early and to those same authors who were patient as others submitted their articles a touch later. I am also very thankful to Joanne Husovski, who pretended not to be annoyed when I would reply to an e-mail four months later as if she had just sent the message. I am most thankful for many of my mentors in the field, especially Dr Vânia Nosé, who have participated in this undertaking, and I look forward to many more productive, collaborative years ahead.

Peter M. Sadow, MD, PhD Pathology Service Massachusetts General Hospital Department of Pathology Harvard Medical School 55 Fruit Street Boston, MA 02114, USA

E-mail address: psadow@mgh.harvard.edu 